detected since. But 50 years on, physicists still have no idea how nature accelerates elementary particles to such high energies. The energies far exceed the range of Earthbound accelerators such as the Large Hadron Collider (LHC) near Geneva, Switzerland; mimicking them would require a ring the size of Earth's orbit around the Sun.

There is also much we need to find out about neutrinos themselves - their accurate masses, how they transform from one type (flavour) into another, and whether other predicted forms (such as 'sterile' neutrinos) exist. Neutrinos could also help to find dark matter, invisible material that has a part in controlling the motions of stars, gas and galaxies. Decaying or annihilating dark matter could produce energetic neutrinos that would be visible to neutrino telescopes.

The downside of neutrinos' weak interactions is that an enormous detector is required to catch enough particles to distinguish the few space-borne ones from the many more originating from Earth's atmosphere. IceCube is the largest neutrino-detection array in operation but it is too small, and further data collection is probably too slow to yield major breakthroughs in the next decade.

Bigger neutrino observatories, with volumes that are 10-100 times greater than that of IceCube, are essential to explore the most energetic processes in the Universe. Determining the masses of different types of neutrino and studying how neutrinos interact with matter within Earth could distinguish or rule out some models of extra spatial dimensions and address key concerns for highenergy nuclear physics such as the density of gluons (which mediate forces between quarks) in heavy nuclei.

Designs for neutrino telescopes are on the drawing board and could be up and running in five to ten years - if the astro-, particle- and nuclear-physics communities can come together and coordinate funding. A complementary set of several neutrino observatories would test physics at energies beyond the LHC's at a fraction of the cost tens to hundreds of millions, rather than tens of billions, of dollars.

\section{MORE QUESTIONS THAN ANSWERS}

IceCube, which became fully operational in Antarctica in 2010 (and with which I have been involved since 2004), detects blue light: Cherenkov radiation that is emitted by the charged particles produced when energetic neutrinos interact with atomic nuclei in water or ice. Computers comb through the data to look for interactions - long tracks or radial cascades of particles emanating from a point (see 'Neutrino observatory'). IceCube sees more than 50,000 neutrino candidates per year. Fewer than $1 \%$ are from space.

There are several ways to distinguish cosmic from atmospheric neutrinos. The

\section{NEXT-GENERATION NEUTRINO TELESCOPES}

Bigger neutrino arrays have been proposed that would catch enough cosmic neutrinos to probe extreme energies and test basic physics and astronomy.

\begin{tabular}{|c|c|c|c|c|}
\hline Experiment & Detects & Where & Volume & Estimated cost (US\$) \\
\hline IceCube-Gen2 & Optical & South Pole, Antarctica & $10 \mathrm{~km}^{3}$ & $\$ 400$ million \\
\hline $\begin{array}{l}\text { Cubic Kilometre } \\
\text { Neutrino Telescope } \\
\text { (KM3NeT) }\end{array}$ & Optical & $\begin{array}{l}\text { Mediterranean Sea (two } \\
\text { sites being considered) }\end{array}$ & $5 \mathrm{~km}^{3}$ & $\$ 250$ million \\
\hline $\begin{array}{l}\text { Gigaton Volume } \\
\text { Detector }\end{array}$ & Optical & Lake Baikal, Russia & $1 \mathrm{~km}^{3}$ & Unknown \\
\hline Askaryan Radio Array & Radio & South Pole, Antarctica & $>100 \mathrm{~km}^{3}$ & $\begin{array}{l}\$ 5 \text { million to } \\
\$ 30 \text { million }\end{array}$ \\
\hline ARIANNA & Radio & $\begin{array}{l}\text { Ross Ice Shelf, } \\
\text { Antarctica }\end{array}$ & $>100 \mathrm{~km}^{3}$ & $\begin{array}{l}\$ 5 \text { million to } \\
\$ 30 \text { million }\end{array}$ \\
\hline
\end{tabular}

highest-energy events are more likely to be astrophysical. Atmospheric neutrinos are accompanied by particle showers, which can be seen with detectors on the ice surface. Muons, short-lived subatomic particles produced in these showers, are 500,000 times more numerous than neutrinos, and can also penetrate the ice; so signals accompanied by muons travelling downwards from the sky are probably atmospheric in origin. That leaves extremely energetic events with light trails that are travelling upwards (through Earth) or that originate from a point within the array volume as potentially astrophysical in origin.

Since 2010, IceCube has seen about 60 astrophysical neutrino candidates ${ }^{2,3}$. Other experiments are too small to detect any such neutrinos; these include ANTARES, an array of strands of detectors anchored to the floor of the Mediterranean Sea off Marseilles, France, and another similar array in Lake Baikal, Russia. Their detection rate of astrophysical neutrinos is as high as could be expected - if there were more neutrinos, they would drain the cosmic rays of most of their energy ${ }^{4}$. So finding the astrophysical sources of the neutrinos should be easy. The fact that we have not is a growing puzzle.

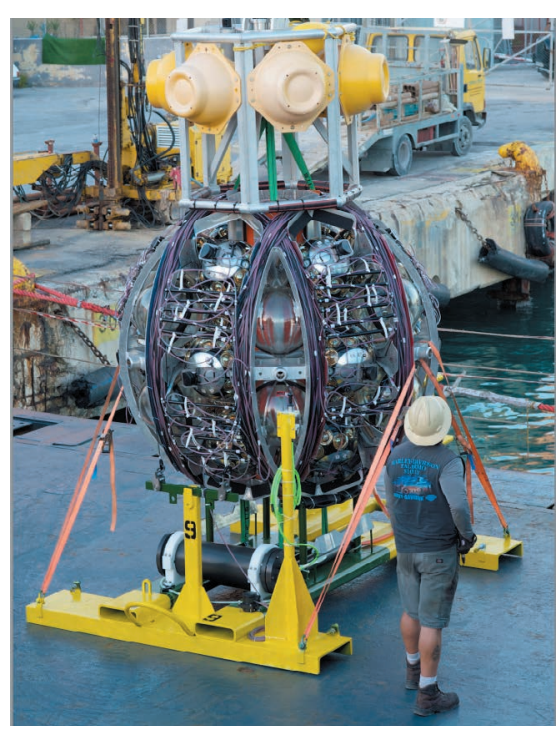

A string of optical modules of the KM3NeT array.
So far, neutrinos do not seem to be coming from particular sites on the $\mathrm{sky}^{5}$, although several groups have suggested a weak link to the plane of the Milky Way. And analyses disfavour the many sites once thought likely to have accelerated energetic cosmic rays and neutrinos, including $\gamma$-ray bursts (GRBs) and active galactic nuclei (AGNs).

GRBs are short bursts of powerful $\gamma$-rays that are picked up by satellites. They are

"Finding the
astrophysical
sources of the
neutrinos should
be easy."
thought to emanate either from a black hole coalescing with a neutron star or another black hole (producing a rapid burst lasting less than 2 seconds); or from the slower collapse of supermassive stars (bursts lasting seconds or minutes). Particles are accelerated by the implosion or explosion. Of more than 800 GRBs examined by IceCube scientists, none was accompanied by a burst of neutrinos, implying that GRBs can produce at most $1 \%$ of the astrophysical neutrinos seen by IceCube ${ }^{6}$.

AGNs are galaxies that at their centres have supermassive black holes accreting gas. Particles may be accelerated to relativistic speeds in jets of material that are blasted out from the black hole. But IceCube sees no associations between energetic neutrinos and active galaxies with jets that point towards Earth, suggesting that active galaxies explain at most $30 \%$ of the neutrinos ${ }^{7}$.

Other unlikely sources include starburst galaxies, which contain dusty regions of intense star formation that are riddled by supernova explosions ${ }^{8}$; magnetars, which are neutron stars surrounded by strong magnetic fields that expel powerful bursts of neutrinos for a few days (these should have been seen by IceCube); and supernova remnants, whose magnetic fields are too weak to explain the most energetic neutrinos ${ }^{9}$, even though they are believed to be responsible for most lower-energy (up to about $10^{16} \mathrm{eV}$ ) cosmic rays seen in the Galaxy.

More exotic possibilities remain untested: as-yet-unseen supermassive dark-matter 
particles that annihilate and produce energetic neutrinos; or the decay of cosmic 'strings', discontinuities in space-time left over from the Big Bang.

IceCube has also tested alternative physics theories. It has constrained how neutrinos 'oscillate' from one flavour to another and set limits on the properties of dark matter and on the constituents of high-energy air showers.

\section{NEXT GENERATION}

There are two ways forward: enlarge the current optical arrays to collect more neutrinos, or find other strategies for isolating the highest energy neutrinos that must be cosmic in origin. These approaches cover different energy ranges and thus complementary physics. Both merit support.

First, larger optical Cherenkov telescopes could be deployed in ice or a lake, sea or ocean - similar to IceCube or ANTARES but with more efficient optical sensors and cheaper technology. Several groups have developed advanced designs for these concepts but lack funding. The detectors could be constructed and operational by the early 2020s. For IceCube, technical improvements would include more efficient drilling technology and sensors that fit in narrower bore holes, which are cheaper to drill.

Different sites offer different benefits. Antarctica offers a large expanse of clear, compacted ice and infrastructure. But arrays in the Northern Hemisphere, for example, in the Mediterranean, can more directly observe astrophysical neutrinos from the centre of the Galaxy that have passed through Earth, without having to reject down-going atmospheric neutrinos, as a southern site would have to. The absence of potassium-40 and the lower bioluminescence in fresh water (which contribute to background light and can confuse the reconstruction of particle tracks), and the presence of a frozen surface during the winter, simplifying construction, make Lake Baikal an attractive site.

The second approach requires catching neutrinos with energies above $10^{8} \mathrm{GeV}$. Neutrinos this energetic are rare - IceCube has seen none - and an array of at least $100 \mathrm{~km}^{3}$ would be needed to capture enough events. Because optical Cherenkov light travels only tens of metres in ice or water, covering such a volume would require millions of sensors and would be expensive.

A more practical way is to search for radio emissions from neutrino interactions with the Antarctic ice sheet. When the neutrinos hit an atomic nucleus in the ice, they create a shower of charged particles that give off radio waves in the 50 megahertz to 1 gigahertz frequency range, as well as visible light. Radio waves can propagate for kilometres through ice. So an radio-sensing array over $100 \mathrm{~km}^{3}$ could be more sparsely populated with instruments, with roughly one station per cubic

\section{NEUTRINO OBSERVATORY}

As a neutrino from space (1) interacts with atomic nuclei in water or ice, the shower of particles given off emit blue light (2). The light can be tracked with detectors to reveal the neutrino's energy and the direction the particle came from (3).

\section{(1) Cosmic neutrino passes through Earth.}

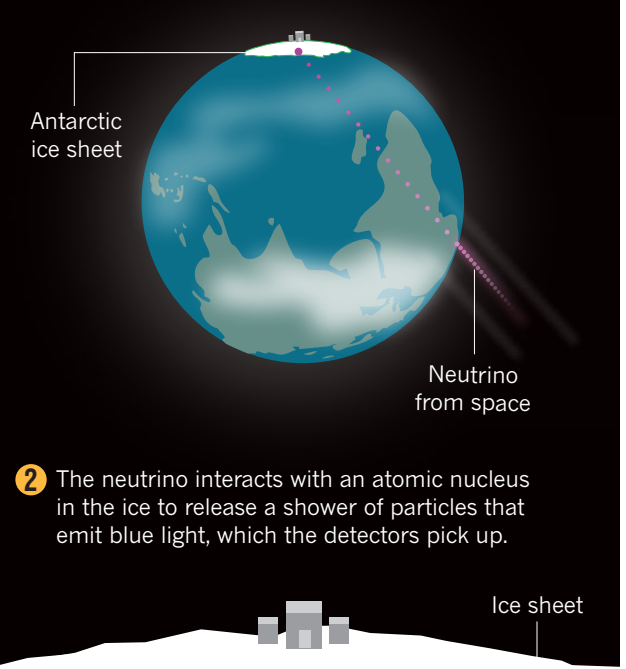

Photon detectors

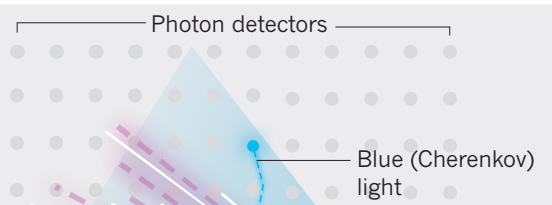
light
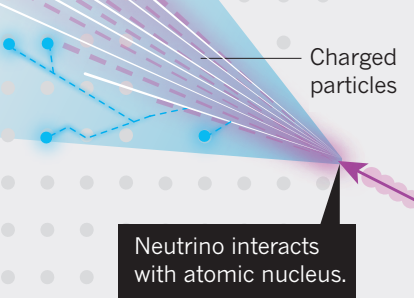

3 Strings of buried optical sensors tracks blue light from the particles as they move through the array.

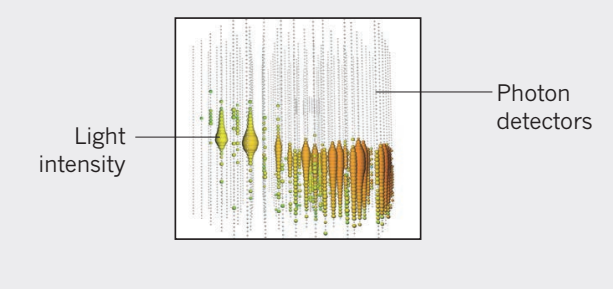

kilometre. The radio pulses from neutrinos with energies above $10^{8} \mathrm{GeV}$ should be strong enough for antennas in the ice to pick up. Two international groups are building prototypes and have sought funding to expand (I am involved with one, ARIANNA).

\section{GREEN LIGHT}

With a range of affordable, next-generation designs shovel-ready, decisions about design priorities need to be made and grants deployed. The main obstacles are limited national science budgets and funding-agency silos. Neutrino astronomy falls between the particle-, nuclear- and astrophysics communities, which need to pool 状 resources to realize the promise of these 0 techniques.

First, one or both of the successors to IceCube and ANTARES should be funded and built. An upgraded IceCube experiment (IceCub-Gen2) and the Cubic Kilometre Neutrino Telescope (KM3NeT), a proposed European project, are both strong candidates (see 'Next-generation neutrino telescopes'). If necessary, the teams coordinating IceCube, KM3NeT and the Gigaton Volume Detector ${ }^{10}$, a proposed Russian array, should explore merging these collaborations to focus on a single large detector at the most cost-effective site. Funding should be sought from a wider range of agencies, including those focused on particle and nuclear physics.

Second, at least one $100-\mathrm{km}^{3}$ radiodetection array needs to get the go ahead. Because such a project can be done only in Antarctica, the onus is on the US National Science Foundation, which is the largest supporter of Antarctic research and realistically the only group that has adequate logistical resources to pull off such a project. Many non-US groups are interested, and collaborations should be set up and costs shared internationally. Once proven, such an array could be expanded to cover $1,000 \mathrm{~km}^{3}$ by around 2030 to monitor the ultra-high-energy Universe.

By finding the astrophysical sources of ultra-energetic neutrinos and cosmic rays — or ruling out remaining models - the next generation of neutrino observatories is guaranteed to make discoveries.

Spencer Klein is a senior scientist in the Nuclear Science Division, Lawrence Berkeley National Laboratory, and a research physicist at the University of California, Berkeley, Berkeley, California, USA.

e-mail:srklein@lbl.gov

1. Halzen, F. \& Klein, S. R, Phys. Today 61N5, 29-35 (2008).

2. Aartsen, M. G. et al. Phys. Rev. Lett. 111, 021103 (2013).

3. IceCube Collaboration. Preprint at https://arxiv.org/abs/1510.05223 (2015)

4. Bahcall, J. \& Waxman, E. Phys. Rev. D 64, 023002 (2001).

5. IceCube Collaboration. Preprint at https://arxiv.org/abs/1510.05222 (2015).

6. Aartsen, M. G. et al. Astrophys. J. 805, L5-L12 (2015).

7. DeYoung, T. EPJ Web Conf. 116, 11004 (2016)

8. Bechtol, K. et al. Preprint at https://arxiv.org/ abs/1511.00688 (2015).

9. Chakraborty, S. \& Izaguirre, I. Phys. Lett. B 745, 35-39 (2015)

10.Avrorin, A. D. et al. Preprint at http://arxiv.org/ abs/1511.02324 (2015). 